\title{
Método de evaluación de las reglas de cambio entre planes de inspección normal y reducida con base en tablas Military Standard 105e
}

\author{
Evaluation method for the switching rules between normal and reduced inspection \\ plans based on Military Standard 105e \\ Nestor Enrique Caicedo Solano ${ }^{1} \quad$ Laura Juliana Mahecha Bernal $^{2}$ \\ Recibido 28 de agosto de 2013, aceptado 29 de mayo de 2014 \\ Received: August 28, 2013 Accepted: May 29, 2014
}

\begin{abstract}
RESUMEN
Las reglas de cambio entre inspección ajustada, normal y reducida propuestas por las tablas Mil-Std105 e, forman parte de los esquemas de muestreo más utilizados en inspección por atributos. Este artículo ilustra un método general que modela las características de las reglas de cambio entre inspección normal y reducida por medio de cadenas de Markov, y facilita el diseño y aplicación de los planes de inspección. Este método generaliza el cambio entre diferentes condiciones de inspección, las que se traducen en estados de Markov.

Los modelos se expresan mediante las curvas características de operación; por tanto, al momento del uso ofrecen la posibilidad de reducir muestras, mejorar certidumbre, disminuir costos y monitorear los diferentes niveles de calidad de productos.
\end{abstract}

Palabras clave: Calidad, muestreo por aceptación, cadenas de Markov, Mil-Std-105e, costos.

\begin{abstract}
Exchange rules between set, normal and reduced inspection, proposed by Mil-Std-105e tables, belong to the most widely used sampling schemes of inspection by attributes. This article illustrates a general method that models the characteristics of the switching rules between regular and reduced inspection through Markov chains and facilitates the design and implementation of inspection plans. This method generalizes the switching between different inspection conditions in Markov states.

The models are expressed through the operating characteristic curves, which allow for the possibility of reducing samples, improving certainty, reducing costs and monitoring of the different levels of quality.
\end{abstract}

Keywords: Quality, acceptance sampling, Markov chains, Mil-Std-105e, costs.

\section{INTRODUCCIÓN}

La inspección por atributos clasifica los lotes conformes o no conformes. Los planes presentados en Military Standard 105e son aplicables a la inspección de componentes y materiales crudos, operaciones y servicios, materiales en proceso, suministros en almacenamiento, operaciones de mantenimiento, datos y procesos administrativos, entre otros [1].

Con la inspección normal se comienza el proceso de inspección, y se cambia entre inspecciones (normal, ajustada y reducida) de acuerdo con las reglas de

1 Departamento de Ingeniería Industrial. Universidad Autónoma del Caribe. Calle 90 \# 46-112. Barranquilla, Colombia. E-mail: nestor.caicedo@uac.edu.co

2 Departamento de Ingeniería Industrial. Universidad de los Andes. Carrera $1 \mathrm{~N}^{\circ} 18^{\mathrm{a}}-12$. Bogotá, Colombia.

E-mail: la-mahec@uniandes.edu.co 
cambio [2], según las condiciones de calidad que los lotes van ofreciendo durante el tiempo. Cuando la calidad del productor es buena históricamente, $\mathrm{y}$ varios lotes pasan la inspección normal, es deseable instaurar la inspección reducida. Esta clase de inspección le permite al productor tener una mayor probabilidad de aceptación del lote, y lo premia por sus buenos niveles de calidad.

El presente estudio ilustra un método de construcción de las curvas características de operación, con el fin de obtener ecuaciones que describan integralmente las probabilidades del sistema de estar en algún estado en particular, de acuerdo con el método propuesto por Hobbs [3], lo que hace de este trabajo una extensión del método. Para este caso se consideran los estados de inspección normal y reducida, y la probabilidad de cambio entre estos. Los principales criterios de evaluación y comparación de las reglas de cambio propuestas son los siguientes:

a. La probabilidad de aceptación en diferentes puntos de la curva característica de operación.

b. La probabilidad de estar en inspección reducida.

c. La probabilidad de cambiar de inspección normal a reducida y viceversa.

d. La probabilidad de estar en inspección normal.

La literatura acerca de muestreo por aceptación e inspección por atributos no es muy extensa. La gran mayoría de los trabajos de evaluación y caracterización fueron desarrollados usando Mil-Std-105d [4]. Estos trabajos también aplican para Mil-Std-105E, que es la última revisión del estándar. Las diferencias entre estos dos esquemas son únicamente cambios editoriales y algunas curvas características de operación adicionales, además de reducir el número de lotes en inspección ajustada de diez a cinco para descontinuar la producción [5].

Hobbs propone el método desarrollado en este estudio, analizando únicamente 2 estados de inspección (normal y ajustada), pero no analiza los 3 estados de inspección en conjunto.

T. Koyama [6], plantea un estudio para las características de cambio entre inspección normal, ajustada y reducida de Mil-Std-105d mediante la teoría de cadenas de Markov y la teoría de gráficos de señales [6-7]. Además presenta una contribución al diseño de nuevas reglas que provean las características que se requieran en el plan de muestreo. En su artículo, T. Koyama [7] expone un estudio de las propiedades transientes de las tablas Mil-Std 105d. Interpreta el comportamiento estadístico de las reglas de cambio mediante el desarrollo de las matrices estocásticas y sus funciones generadoras, se obtiene el cálculo de las probabilidades transitorias de cambio, probabilidades estacionarias, la media y varianza del número de lotes que anteceden el cambio de un estado de inspección a otro. Shilling \& Sheesley [8] se encargaron de analizar el desempeño de las tablas estándar mediante el análisis de las características que comparten las tablas estándar de acuerdo con cada nivel de inspección (normal, ajustada y reducida). Esto, con el fin de demostrar las ventajas del uso apropiado del estándar y los niveles de protección ofrecidos tanto para el consumidor como para el productor, mediante el análisis detallado de las curvas características de operación y otros parámetros [9]. Además, proporcionan un análisis del mejoramiento del nivel de calidad aceptable (NCA), porcentaje de producto no conforme presente en un lote, y de la calidad saliente promedio (CSP), los cuales son criterios claves para la evaluación de los planes de muestreo.

Brugger [10] propone un método simplificado para el modelamiento de la regla propuesta por la ANSI/ ASQC Z1.4 sin números límites. Presenta una estructuración más sencilla de los estados posibles durante el proceso de inspección. Chakraborty [11] demuestra que debido al reducido tamaño de los estados, el número de ecuaciones por resolver es menor y los posibles errores algebraicos se disminuyen.

Yang [12] presenta una forma innovadora de ver las reglas de cambio propuestas en Mil-Std-105d, expresando la calidad saliente promedio (CSP) en términos de los momentos de los tiempos de parada durante el proceso de inspección. Estos tiempos de parada se definen como el número de lotes inspeccionados bajo un determinado plan antes de cambiar a otro diferente.

Un estudio más reciente es el desarrollado por Shmueli \& Cohen [13] que estudiaron las distribuciones de probabilidad que son frecuentemente encontradas en el muestreo por aceptación. Estos autores presentan un método de aproximación para la derivación de la función de densidad de probabilidad, basados en 
la expansión de fracciones parciales, con el fin de derivar la función de probabilidad exacta de múltiples variables a partir de su función generadora. Presentan también una aplicación al esquema de muestreo de Mil-Std-105d y las reglas de cambio mediante una aproximación teórica a las propiedades del estándar usando distribuciones de probabilidad para la regla de paso de inspección ajustada a normal. Proponen estos análisis, ya que argumentan que las reglas de cambio propuestas por el estándar no están basadas en su estructura probabilística, sino en juicios empíricos.

\section{METODOLOGÍA}

El comportamiento de los lotes durante el proceso de inspección bajo una determinada regla, puede representarse gráficamente como una serie de nodos conectados entre sí por flechas. Estos nodos representan los estados probables de inspección durante el proceso [12] y continuar en ellos, o el cambio entre planes según el comportamiento histórico de la calidad del producto en el tiempo, como ilustra la Figura 1.

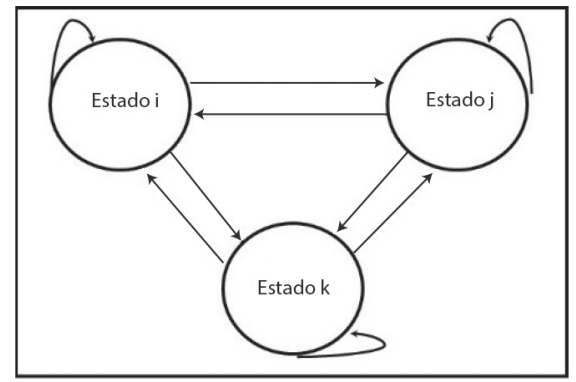

Figura 1. Diagrama de estados.

Las flechas representan las probabilidades de pasar de un estado a otro. Esta representación gráfica se conoce como una cadena de Markov y define un proceso markoviano finito, el cual tiene asociada su correspondiente matriz estocástica.

Una cadena de Markov puede describir un proceso en el cual se puede ir de un estado a otro, de tal forma que la probabilidad de ir de uno a los demás estados depende del hecho que esté en el estado actual, y no depende de su estancia en estados previos o posteriores [10]. Una vez se tiene la matriz estocástica correspondiente, se procede a calcular el vector único de probabilidad para resolver el sistema de ecuaciones y ejecutar los cálculos necesarios para la evaluación de las reglas propuestas. A continuación se presentan las reglas de cambio entre inspección normal y reducida a desarrollar en el presente trabajo:

Regla 1: Usar inspección reducida si tres lotes han sido aceptados bajo inspección normal. Usar inspección normal si un lote ha sido rechazado bajo inspección reducida.

Regla 2: Usar inspección reducida si cinco lotes han sido aceptados bajo inspección normal. Usar inspección normal si un lote ha sido rechazado bajo inspección reducida.

Regla 3: Usar inspección reducida si siete lotes han sido aceptados bajo inspección normal. Usar inspección normal si un lote ha sido rechazado bajo inspección reducida.

Regla 4: Regla ANSI/ASQC Z1.9 (1980) y ANSI/ ASQC Z1.4 (1981). Usar inspección reducida si diez lotes han sido aceptados bajo inspección normal. Usar inspección normal si un lote ha sido rechazado bajo inspección reducida.

\section{GRAFOS, MATRICES ESTOCÁSTICAS Y ECUACIONES}

Para el desarrollo de las reglas se usará la notación presentada en la ecuación planteada por Hobbs [3], siendo:

$\mathrm{a}=$ Probabilidad de aceptación bajo inspección Normal.

$\mathrm{c}=$ Probabilidad de aceptación bajo inspección Reducida.

$\mathrm{n}=\mathrm{n}$-ésima regla propuesta para los cambios de inspección.

$\mathrm{P}(\mathrm{CO}) \mathrm{n}=$ Probabilidad de aceptación de acuerdo con la regla para inspección Normal y Reducida para la n-ésima regla.

$\mathrm{P}(\mathrm{R}) \mathrm{n}=$ Probabilidad de permanecer en inspección reducida para la n-ésima regla.

$\mathrm{P}(\mathrm{NaR}) \mathrm{n}=$ Probabilidad de cambiar de inspección normal a reducida o viceversa para la n-ésima regla. $\mathrm{P}(\mathrm{N}) \mathrm{n}=$ Probabilidad de estar en inspección normal para la n-ésima regla. Este comportamiento estocástico se ilustra desde la Figura 2 hasta la Figura 5, mostrado como matrices según las reglas de cambio, Regla 1 ilustrada en la Tabla 1, Regla 2 
ilustrada en la Tabla 2, Regla 3 ilustrada en la Tabla 3, igualmente la Regla 4 ilustrada en la Tabla 4.

\section{Regla 1}

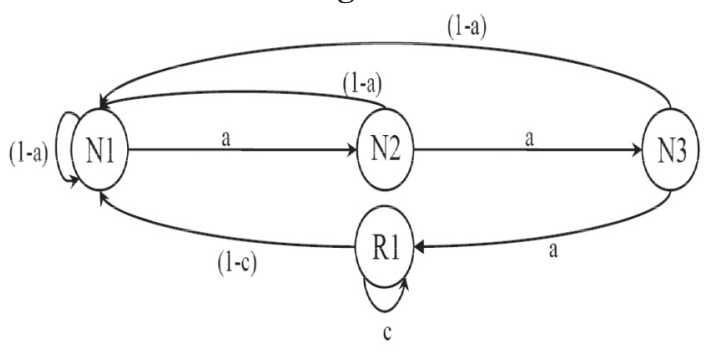

Figura 2. Grafo regla 1.

Tabla 1. Matriz estocástica regla 1.

\begin{tabular}{|c|c|c|c|c|c|}
\hline & i & N1 & N2 & N3 & R1 \\
\hline \multirow{3}{*}[R]{$=$} & N1 & $(1-a)$ & a & - & - \\
\cline { 2 - 6 } & N2 & $(1-a)$ & - & a & - \\
\cline { 2 - 6 } & N3 & (1-a) & - & - & a \\
\cline { 2 - 6 } & R1 & (1-c) & - & - & $c$ \\
\hline
\end{tabular}

$$
P(C O)_{1}=\frac{a\left[1+a+a^{2}-(1-a) c\right]}{\left[1+a+a^{2}+a^{3}\right]-\left[\left(1+a+a^{2}\right) c\right]}
$$

$$
P(R)_{1}=\frac{a^{3} c}{\left.\left[1+a+a^{2}+a^{3}\right]-\left(1+a+a^{2}\right) c\right]}
$$

$$
P(N a R)_{1}=\frac{-a^{3}(c-1)}{\left.\left[1+a+a^{2}+a^{3}\right]-\left(1+a+a^{2}\right) c\right]}
$$

$$
P(N)_{1}=\frac{\left(a^{3}-a^{2}-a-1\right)-(c-1)}{\left.\left[1+a+a^{2}+a^{3}\right]-\left(1+a+a^{2}\right) c\right]}
$$

\section{Regla 2}

\begin{tabular}{|c|c|c|c|c|c|c|c|}
\hline & & N1 & $\mathrm{N} 2$ & N3 & N4 & N5 & $\mathrm{R} 1$ \\
\hline \multirow{6}{*}[R]{$=$} & N1 & $(1-a)$ & $\mathrm{a}$ & - & - & - & - \\
\hline & $\mathrm{N} 2$ & $(1-a)$ & - & $\mathrm{a}$ & - & - & - \\
\hline & N3 & $(1-a)$ & - & - & $\mathrm{a}$ & - & - \\
\hline & N4 & $(1-a)$ & - & - & - & $\mathrm{a}$ & - \\
\hline & N5 & (1-a) & - & - & - & - & $\mathrm{a}$ \\
\hline & $\mathrm{R} 1$ & $(1-c)$ & - & - & - & - & $\mathrm{c}$ \\
\hline
\end{tabular}

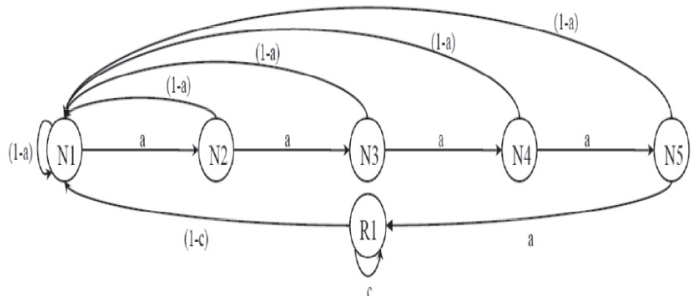

Figura 3. Grafo regla 2.
Tabla 2. Matriz estocástica regla 2.

$$
P(C O)_{2}=\frac{a\left(1+\sum_{i=1}^{4} a^{i}-\left[\left(1+\sum_{i=1}^{3} a^{i}\right) c\right]\right.}{\left[1+\left(\sum_{i=1}^{5} a^{i}\right)\right]-\left(1+\left(\sum_{i=1}^{4} a^{i}\right) c\right.}
$$

$$
P(R)_{2}=\frac{a^{5} c}{\left[1+\left(\sum_{i=1}^{5} a^{i}\right)\right]-\left(1+\left(\sum_{i=1}^{4}\right) a^{i}\right) c}
$$

$$
\begin{gathered}
P(N a R)_{2}=\frac{-a^{5}(c-1)}{\left[1+\left(\sum_{i=1}^{5} a^{i}\right)\right]-\left(1+\left(\sum_{i=1}^{4} a^{i}\right) c\right.} \\
P(N)_{2}=\frac{-\left(a^{5}-a^{4}-a^{3}-a^{2}-a-1\right)(c-1)}{\left[1+\left(\sum_{i=1}^{5} a^{i}\right)\right]-\left(1+\left(\sum_{i=1}^{4} a^{i}\right) c\right]}
\end{gathered}
$$

\begin{tabular}{|c|c|c|c|c|c|c|c|c|c|}
\hline & $\mathrm{i}$ & N1 & $\mathrm{N} 2$ & N3 & N4 & N5 & N6 & N7 & R1 \\
\hline \multirow{8}{*}[R]{$=$} & $\mathrm{N} 1$ & $(1-a)$ & $\mathrm{a}$ & - & - & - & - & - & - \\
\hline & $\mathrm{N} 2$ & $(1-a)$ & - & $\mathrm{a}$ & - & - & - & - & - \\
\hline & N3 & $(1-a)$ & - & - & $\mathrm{a}$ & - & - & - & - \\
\hline & $\mathrm{N} 4$ & $(1-a)$ & - & - & - & $\mathrm{a}$ & - & - & - \\
\hline & N5 & $(1-a)$ & - & - & - & - & $\mathrm{a}$ & - & - \\
\hline & N6 & (1-a) & - & - & - & - & - & $\mathrm{a}$ & - \\
\hline & N7 & (1-a) & - & - & - & - & - & - & $\mathrm{a}$ \\
\hline & R1 & $(1-c)$ & - & - & - & - & - & - & c \\
\hline
\end{tabular}

\section{Regla 3}

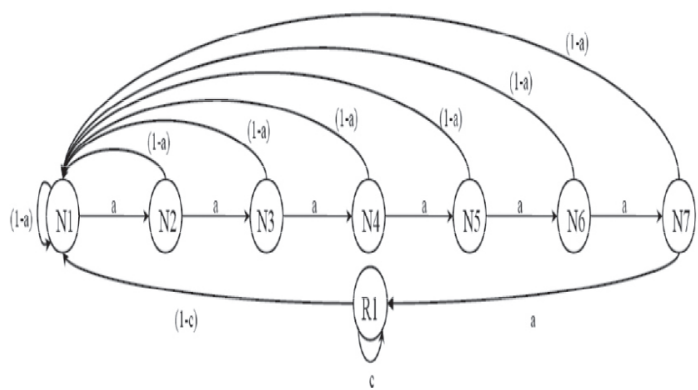

Figura 4. Grafo regla 3.

Tabla 3. Matriz estocástica regla 3. 


$$
\begin{gathered}
P(C O)_{3}=\frac{a\left(1+\sum_{i=1}^{6} a^{i}\right)-\left[\left(1+\sum_{i=1}^{5} a^{i}\right) c\right]}{\left[1+\left(\sum_{i=1}^{7} a_{i}\right)\right]-\left(1+\left(\sum_{i=1}^{6} a_{i}\right) c\right.} \\
P(R)_{3}=\frac{a^{7} c}{\left[1+\left(\sum_{i=1}^{7} a^{i}\right)\right]-\left(1+\left(\sum_{i=1}^{6} a^{i}\right) c\right.} \\
P(N a R)_{3}=\frac{-a^{7}(c-1)}{\left[1+\left(\sum_{i=1}^{7} a^{i}\right)\right]-\left(1+\left(\sum_{i=1}^{6} a^{i}\right) c\right.} \\
P(N)_{3}=\frac{\left(a^{7}-a^{6}-a^{5}-a^{4}-a^{3}-a^{2}-a-1\right)(c-1)}{\left[1+\left(\sum_{i=1}^{7} a^{i}\right)\right]-\left(1+\left(\sum_{i=1}^{6} a^{i}\right) c\right]}
\end{gathered}
$$

\begin{tabular}{|c|c|c|c|c|c|c|c|c|c|c|c|c|}
\hline & $i^{\mathrm{j}}$ & N1 & $\mathrm{N} 2$ & N3 & N4 & N5 & N6 & $\mathrm{N} 7$ & N8 & N9 & N10 & $\mathrm{R} 1$ \\
\hline \multirow{11}{*}[R]{$=$} & N1 & $(1-a)$ & $\mathrm{a}$ & - & - & - & - & - & - & - & - & - \\
\hline & $\mathrm{N} 2$ & $(1-a)$ & - & $\mathrm{a}$ & - & - & - & - & - & - & - & - \\
\hline & N3 & $(1-a)$ & - & - & $\mathrm{a}$ & - & - & - & - & - & - & - \\
\hline & N4 & $(1-a)$ & - & - & - & $\mathrm{a}$ & - & - & - & - & - & - \\
\hline & N5 & $(1-a)$ & - & - & - & - & $\mathrm{a}$ & - & - & - & - & - \\
\hline & N6 & $(1-a)$ & - & - & - & - & - & $\mathrm{a}$ & - & - & - & - \\
\hline & N7 & $(1-a)$ & - & - & - & - & - & - & $\mathrm{a}$ & - & - & - \\
\hline & N8 & $(1-a)$ & - & - & - & - & - & - & - & $\mathrm{a}$ & - & - \\
\hline & N9 & $(1-a)$ & - & - & - & - & - & - & - & - & $\mathrm{a}$ & - \\
\hline & $\mathrm{N} 10$ & $(1-a)$ & - & - & - & - & - & - & - & - & - & $\mathrm{a}$ \\
\hline & $\mathrm{R} 1$ & $(1-c)$ & - & - & - & - & - & - & - & - & - & $\mathrm{c}$ \\
\hline
\end{tabular}

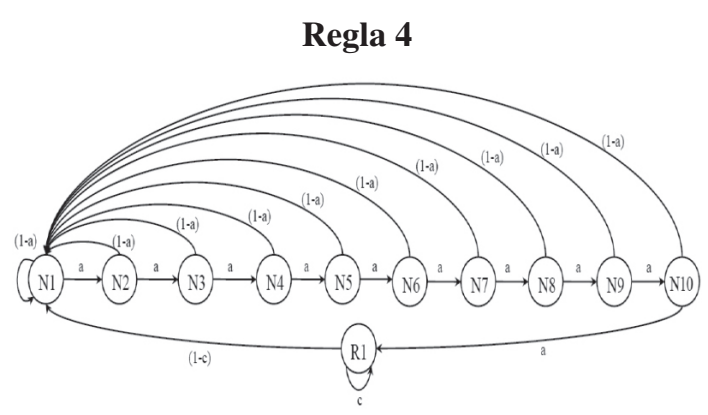

Figura 5. Grafo regla 4.

Tabla 4. Matriz estocástica regla 4.

$P(C O)_{4}=\frac{a\left(1+\sum_{i=1}^{9} a_{i}\right)-\left[\left(1+a+a^{2}\right)\left(1+a^{3}+a^{6}\right) c\right]}{\left[1+\left(\sum_{i=1}^{10} a^{i}\right)\right]-\left[\left(1+a\left(1+a+a^{2}\right)\left(1+a^{3}+a^{6}\right) c\right]\right.}$

$$
P(R)_{4}=\frac{a^{10} c}{\left[1+\left(\sum_{i=1}^{10} a^{i}\right)\right]-\left[\left(1+a+a^{2}\right)\left(1+a^{3}+a^{6}\right) c\right]}
$$

$P(N a R)_{4}=\frac{-a^{10}(c-1)}{\left[1+\left(\sum_{i=1}^{10} a^{i}\right)\right]-\left[\left(1+a\left(1+a+a^{2}\right)\left(1+a^{3}+a^{6}\right) c\right]\right.}$

$$
\begin{aligned}
& P(N)_{4}= \\
& \frac{\left(a^{10}-a^{9}-a^{8}-a^{7}-a^{6}-a^{5}-a^{4}-a^{3}-a^{2}-a-1\right)(c-1)}{\left[1+\left(\sum_{i=1}^{10} a^{i}\right)\right]-\left[\left(1+a\left(1+a+a^{2}\right)\left(1+a^{3}+a^{6}\right) c\right]\right.}
\end{aligned}
$$

\section{Aplicación de las reglas al plan de inspección}

Para el uso de las reglas, se asume que hay un proceso de producción continuo y el tamaño del lote es lo suficientemente grande respecto de la muestra, con el objetivo de reproducir curvas de operación características, mediante la distribución binomial, sabiendo que se conoce el tamaño muestral n, y la proporción de producto defectuoso $\mathrm{p}$, que considera los parámetros para un plan de inspección en calidad.

$$
P(x)=\left(\begin{array}{l}
n \\
x
\end{array}\right) p^{x}(1-p)^{n-x}
$$

Con $\mathrm{p}=0,01, \mathrm{n}=800$ y $\mathrm{x}=14$ para inspección normal y $\mathrm{n}=315$ y $\mathrm{x}=7$ para inspección reducida (Mil-Std-105e), se calculan las probabilidades de aceptación en un rango de NCA de $1 \%$ a 2,6\%. Los valores presentados en la Tabla 4 se usan para la construcción de las curvas características de operación para inspección normal y reducida, que definen los límites de las curvas características de operación de las reglas establecidas. Los valores de a y c pueden ser sustituidos en las fórmulas (10) y (11) para determinar las curvas características de operación compuestas, la probabilidad de estar en inspección reducida, la probabilidad de pasar de inspección normal a reducida y viceversa, y la probabilidad de estar en inspección normal.

\section{Comparación de las curvas características de operación}

Con un nivel de calidad aceptable (NCA) de 1\%, la curva característica de operación deseada es aquella que defina la probabilidad de aceptar o rechazar el lote que contenga un porcentaje de defectuosos mayor al NCA propuesto, lo cual sería una línea vertical en 1,1, ilustrado en la Figura 10. Teniendo en cuenta este criterio, se puede ver que la curva que discrimina el sometimiento de lotes en este punto es la curva característica de operación para la regla 4, dada la alta probabilidad de aceptar el lote respecto de las curvas de otras reglas. Adicionalmente, esta curva es la más próxima a estar en la inspección normal, ya que es necesaria la aceptación de 10 lotes para poder pasar al único estado de inspección reducida. 


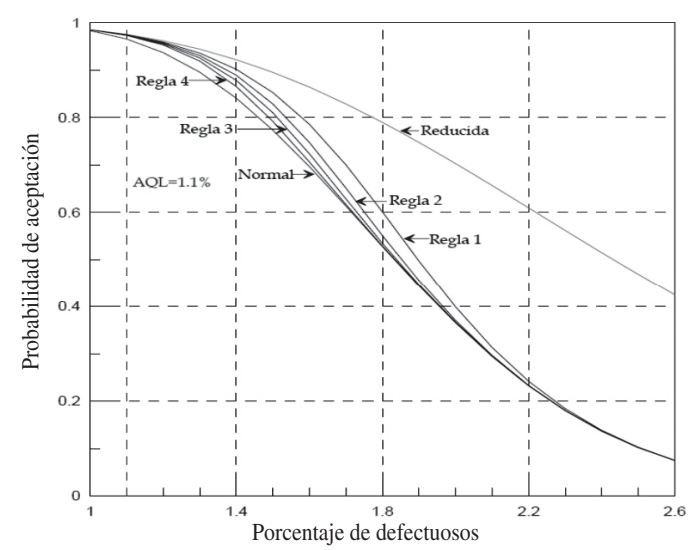

Figura 6. Curvas características de operación.

Tabla 5. Probabilidad de aceptación curvas características de operación.

\begin{tabular}{|c|c|c|c|c|c|c|}
\hline $\begin{array}{c}\text { Porcentaje } \\
\text { de } \\
\text { defectuosos }\end{array}$ & Normal & Reducida & Regla 1 & Regla 2 & Regla 3 & Regla 4 \\
\hline 1,0 & 0,983 & 0,985 & 0,985 & 0,985 & 0,985 & 0,985 \\
1,1 & 0,965 & 0,975 & 0,975 & 0,974 & 0,974 & 0,973 \\
1,2 & 0,937 & 0,962 & 0,959 & 0,957 & 0,955 & 0,953 \\
1,3 & 0,895 & 0,944 & 0,936 & 0,930 & 0,926 & 0,919 \\
1,4 & 0,841 & 0,922 & 0,902 & 0,889 & 0,878 & 0,865 \\
1,5 & 0,773 & 0,895 & 0,853 & 0,829 & 0,810 & 0,792 \\
1,6 & 0,696 & 0,864 & 0,786 & 0,747 & 0,723 & 0,706 \\
1,7 & 0,613 & 0,829 & 0,700 & 0,651 & 0,628 & 0,617 \\
1,8 & 0,528 & 0,790 & 0,601 & 0,551 & 0,534 & 0,529 \\
1,9 & 0,444 & 0,747 & 0,497 & 0,455 & 0,446 & 0,444 \\
2,0 & 0,366 & 0,703 & 0,399 & 0,370 & 0,366 & 0,366 \\
2,1 & 0,295 & 0,656 & 0,313 & 0,296 & 0,295 & 0,295 \\
2,2 & 0,233 & 0,609 & 0,242 & 0,233 & 0,233 & 0,233 \\
2,3 & 0,180 & 0,562 & 0,184 & 0,180 & 0,180 & 0,180 \\
2,4 & 0,137 & 0,515 & 0,138 & 0,137 & 0,137 & 0,137 \\
2,5 & 0,102 & 0,469 & 0,103 & 0,102 & 0,102 & 0,102 \\
2,6 & 0,075 & 0,425 & 0,075 & 0,075 & 0,075 & 0,075 \\
\hline
\end{tabular}

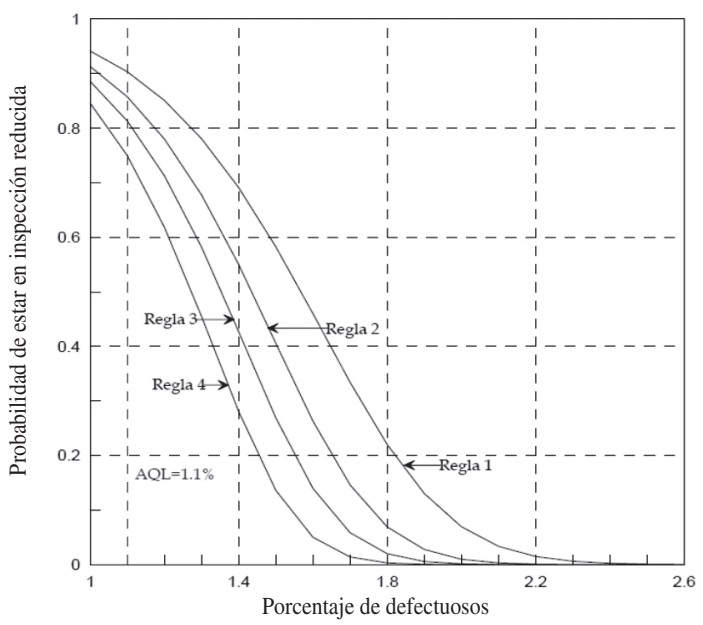

Figura 7. Probabilidad de estar en inspección reducida.
La Figura 7 ilustra el comportamiento de los porcentajes de defectuosos, teniendo en cuenta la probabilidad de encontrarse en el plan de inspección reducida, el nivel de calidad aceptable y las diferentes reglas que rigen los cambios de planes de muestreo según la guía military standard 105E [4-5], necesaria para evaluar atributos.

Tabla 6. Probabilidad de estar en inspección reducida.

\begin{tabular}{|c|c|c|c|c|}
\hline $\begin{array}{c}\text { Porcentaje de } \\
\text { defectuosos }\end{array}$ & Regla 1 & Regla 2 & Regla 3 & Regla 4 \\
\hline 1,0 & 0,941 & 0,913 & 0,886 & 0,846 \\
1,1 & 0,903 & 0,858 & 0,813 & 0,750 \\
1,2 & 0,851 & 0,780 & 0,713 & 0,618 \\
1,3 & 0,781 & 0,678 & 0,582 & 0,455 \\
1,4 & 0,691 & 0,550 & 0,427 & 0,281 \\
1,5 & 0,581 & 0,406 & 0,269 & 0,136 \\
1,6 & 0,460 & 0,263 & 0,140 & 0,050 \\
1,7 & 0,334 & 0,146 & 0,059 & 0,014 \\
1,8 & 0,220 & 0,069 & 0,020 & 0,003 \\
1,9 & 0,130 & 0,028 & 0,006 & 0,000 \\
2,0 & 0,069 & 0,010 & 0,001 & 0,000 \\
2,1 & 0,034 & 0,003 & 0,000 & 0,000 \\
2,2 & 0,015 & 0,001 & 0,000 & 0,000 \\
2,3 & 0,006 & 0,000 & 0,000 & 0,000 \\
2,4 & 0,002 & 0,000 & 0,000 & 0,000 \\
2,5 & 0,001 & 0,000 & 0,000 & 0,000 \\
2,6 & 0,000 & 0,000 & 0,000 & 0,000 \\
\hline
\end{tabular}

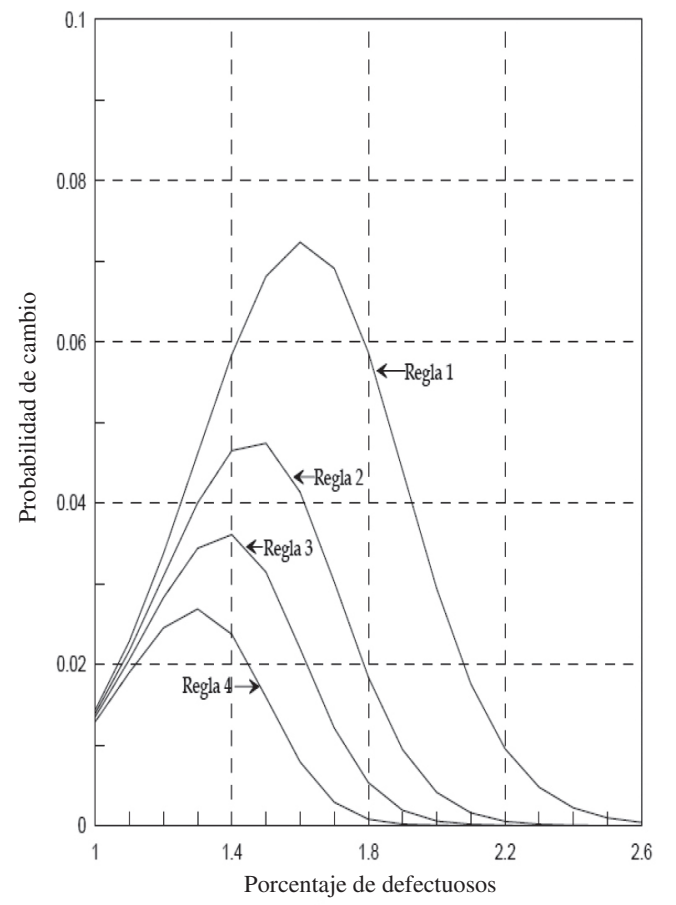

Figura 8. Probabilidad de pasar de inspección normal a reducida. 
Tabla 7. Probabilidad de pasar de inspección normal a reducida y viceversa.

\begin{tabular}{|c|c|c|c|c|}
\hline $\begin{array}{c}\text { Porcentaje de } \\
\text { defectuosos }\end{array}$ & Regla 1 & Regla 2 & Regla 3 & Regla 4 \\
\hline 1,0 & 0,014 & 0,014 & 0,013 & 0,013 \\
1,1 & 0,023 & 0,022 & 0,021 & 0,019 \\
1,2 & 0,034 & 0,031 & 0,028 & 0,025 \\
1,3 & 0,046 & 0,040 & 0,034 & 0,027 \\
1,4 & 0,058 & 0,047 & 0,036 & 0,024 \\
1,5 & 0,068 & 0,047 & 0,031 & 0,016 \\
1,6 & 0,072 & 0,041 & 0,022 & 0,008 \\
1,7 & 0,069 & 0.030 & 0,012 & 0,003 \\
1,8 & 0,059 & 0,018 & 0,005 & 0,001 \\
1,9 & 0,044 & 0,009 & 0,002 & 0,000 \\
2,0 & 0,029 & 0,004 & 0,001 & 0,000 \\
2,1 & 0,018 & 0,002 & 0,000 & 0,000 \\
2,2 & 0,010 & 0,001 & 0,000 & 0,000 \\
2,3 & 0,005 & 0,000 & 0,000 & 0,000 \\
2,4 & 0,002 & 0,000 & 0,000 & 0,000 \\
2,5 & 0,001 & 0,000 & 0,000 & 0,000 \\
2,6 & 0,000 & 0,000 & 0,000 & 0,000 \\
\hline
\end{tabular}

\section{Probabilidad de estar en inspección reducida}

La Figura 7 muestra las condiciones bajo las cuales los lotes sometidos a inspección pueden encontrarse o no en inspección reducida.

La condición de preferencia en este caso sería que los lotes tuvieran baja probabilidad de estar en inspección reducida cuando excede el nivel de calidad aceptable; es decir, cuando el porcentaje de defectuosos sea $1 \%$ o más.

Esta importante diferencia se puede reconocer en la Figura 8, donde se observa que la menor probabilidad de estar en inspección reducida una vez excedido el NCA corresponde a la regla 4.

\section{Probabilidad de pasar de inspección normal a inspección reducida y viceversa}

De acuerdo con Hobbs [3], el desempeño de cada regla en particular está definido por la probabilidad de estar en cualquier estado (para este caso en inspección normal o en inspección reducida), y la probabilidad de pasar entre estados, lo cual se encuentra dado por la siguiente ecuación:

$$
P(N)+P(R)+2 P(N a R)=1
$$

La condición que se prefiere, es que la curva característica de operación tienda a estar sobre la curva para inspección reducida cuando el porcentaje de defectuosos se encuentre por debajo del NCA de $1 \%$, y que esté muy cercana a la inspección normal cuando se encuentre por encima de este nivel de calidad aceptable, para este caso el mejor ejemplo es la regla 4, la cual muestra la mayor probabilidad de aceptar el lote según el porcentaje de no conformidad.

La probabilidad de cambio entre los estados de inspección debe tener un rango estrecho de distribución cuando el NCA es excedido (para este caso que se encuentre por encima de $1 \%$ ). Se puede ver que la regla que más se ajusta a esta condición es la regla 4 , que presenta la menor probabilidad debido al número de lotes que son sometidos a inspección normal para poder pasar a inspección reducida.

Probabilidad de estar en inspección normal y en inspección reducida vs. probabilidad de cambio Con el fin de presentar más claramente la evaluación de los planes de muestreo de acuerdo con las reglas de cambio, a continuación se presentan gráficas para las últimas dos reglas con la probabilidad de estar en inspección normal, en inspección reducida, y la probabilidad de cambio entre ambos estados de inspección. Las Figuras 9 y 10 presentan las variaciones de las probabilidades anteriormente mencionadas para las 2 reglas discriminatorias.

En la gráfica correspondiente a la regla 3 se puede ver que la probabilidad de estar en inspección reducida es alta cuando el nivel de calidad aceptable se encuentra en $1 \%$. Asimismo, se observa que la probabilidad de estar en inspección normal en este mismo punto es muy baja, lo cual es una condición favorable para el productor.

Teniendo en cuenta la interpretación anterior, se observa que la regla que satisface en mayor medida la condición de que no se puedan realizar cambios injustificados, al pasar de inspección normal a reducida es la regla 4 , que se puede evidenciar en las Figuras 9 y 10. Por otro lado, se garantiza una mayor protección al consumidor al aplicar el plan de muestreo con el mayor poder discriminatorio, ya que cuando el nivel de calidad aceptable se excede, la probabilidad de estar en inspección normal aumenta mientras sucede lo contrario con la probabilidad de estar en inspección reducida. Las curvas de operación de las Figuras 9 y 10 ilustran los porcentajes de defectuosos respecto de las reglas de cambio 3 y 4 , respectivamente. 


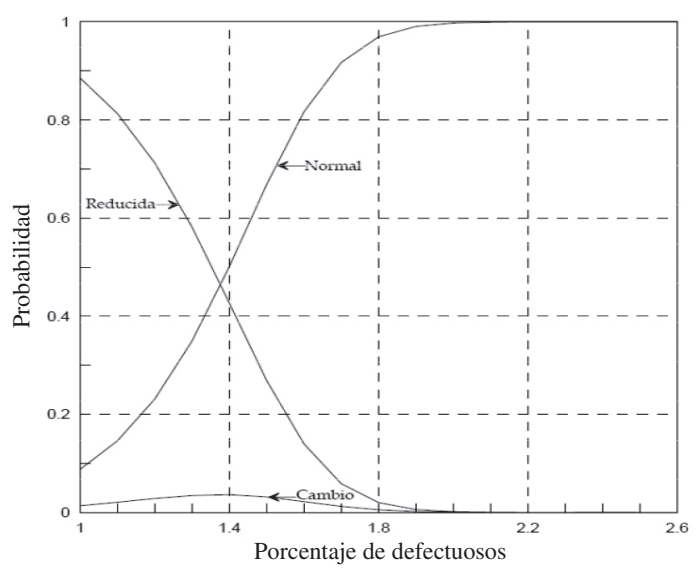

Figura 9. Porcentaje de defectuosos Vs. probabilidades de cambio regla 3 .

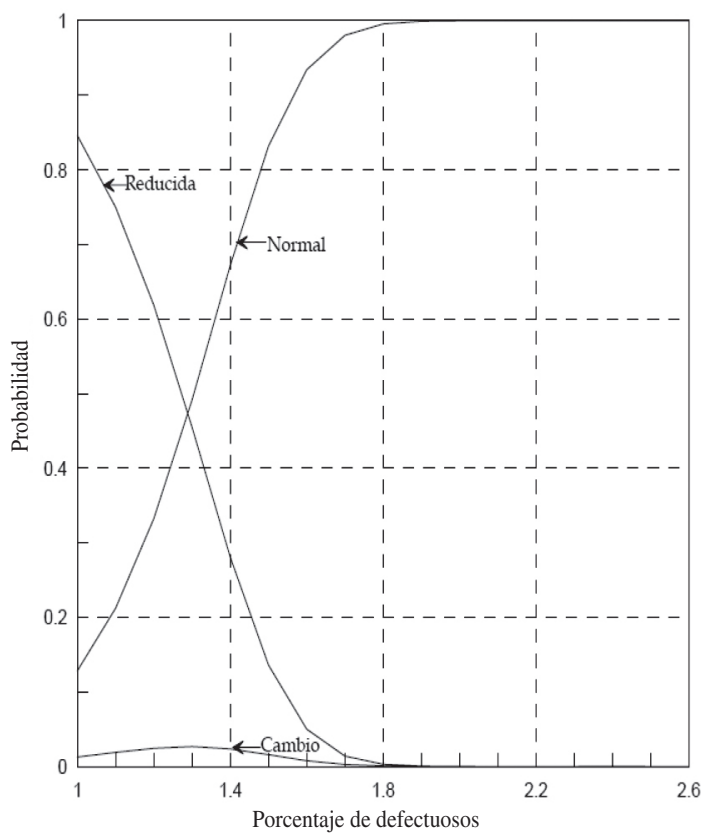

Figura 10.Porcentaje de defectuosos Vs. probabilidades de cambio regla 4.

\section{RESULTADOS}

Con el desarrollo del método para pasar de inspección normal a reducida se observaron una serie de características en los resultados del cálculo de las probabilidades de los criterios necesarios para evaluar los planes de muestreo. Estas características pueden ser generalizadas de la siguiente forma, teniendo en cuenta que solamente hay un estado de inspección reducida permitido por Military Standard 105E.
Nomenclatura:

$\mathrm{S}=$ Número de lotes a someter en inspección normal. $\mathrm{a}=$ Probabilidad de aceptación bajo inspección reducida.

$\mathrm{c}=$ Probabilidad de aceptación bajo inspección normal.

$\mathrm{P}(\mathrm{CO}) \mathrm{n}=$ Probabilidad de aceptación de acuerdo con la regla de cambio de inspección normal a reducida. $\mathrm{P}(\mathrm{R}) \mathrm{n}=$ Probabilidad de permanecer en inspección reducida para cada regla.

$\mathrm{P}(\mathrm{NaR}) \mathrm{n}=$ Probabilidad de cambiar de inspección normal a reducida o viceversa para cada regla.

$\mathrm{P}(\mathrm{N}) \mathrm{n}=$ Probabilidad de estar en inspección normal para cada regla.

$$
\begin{gathered}
P(C O)_{n}=\frac{\left.a\left(\sum_{i=0}^{s-1}\right) a^{i}-c \sum_{i=0}^{s-2} a^{i}\right)}{\left(\sum_{i=0}^{s} a^{i}\right)-\left(c \sum_{i=0}^{s-1} a^{i}\right)} \\
P(R)_{n}=\frac{a^{s} c}{\left(\sum_{i=0}^{s} a^{i}\right)-\left(c \sum_{i=0}^{s-1} a^{i}\right)} \\
P(N a R)=\frac{a^{s}(c-1)}{\left(\sum_{i=0}^{s} a^{i}\right)-\left(c \sum_{i=0}^{s-1} a^{i}\right)} \\
P(N)_{n}=\frac{(c-1)\left(a^{s}-\sum_{i=0}^{s-1} a^{i}\right)}{\left(\sum_{i=0}^{s} a^{i}\right)-\left(c \sum_{i=0}^{s-1} a^{i}\right)}
\end{gathered}
$$

La generalización de este método permite obviar los cálculos correspondientes al desarrollo de las reglas de cambio entre inspecciones, ya que estos se vuelven dispendiosos al incrementar el número de lotes que deben ser aceptados bajo inspección normal.

\section{CONCLUSIONES}

Las reglas de cambio que se usen al momento de implementar un plan de muestreo en el proceso de inspección, usando sistemas de muestreo como Mil-Std-105e, son los criterios más importantes al momento de usar estos sistemas de muestreo.

La exclusión de la inspección reducida de los planes de muestreo a los que puede acceder el productor, lo priva de las recompensas que puede obtener si garantiza una calidad excepcional en sus productos. El acceso a la inspección reducida es la 
recompensa que recibe el productor por su buena calidad, permitiendo una menor cantidad de tamaño de muestra y mayores probabilidades de aceptación del lote. Esta clase de inspección debe ser tenida en cuenta en los planes de muestreo que se adopten en la inspección por atributos de los productos.

El diseño de diferentes reglas de cambio entre clases de inspecciones puede efectuarse según estipulaciones de cada una de las partes. El desempeño de estas reglas está reflejado en las curvas características de operación, sin embargo, este no es el único criterio de evaluación de estas al momento de decidir cuál regla de cambio es mejor. Se debe tener en cuenta las demás curvas que se derivan del desarrollo del método presentado en este trabajo, como son: la probabilidad de estar en inspección normal, la probabilidad de estar en inspección reducida, la probabilidad de cambio entre inspecciones.

A futuro, estos planes de muestreo podrán integrarse a los indicadores de capacidad de procesos, teniendo en cuenta que estos indicadores son significativos para la toma de decisiones en calidad de productos y podrán determinar el cambio de un plan de muestreo según sus resultados. En cuanto a su posible campo de aplicación, el presente trabajo propicia un espacio para la investigación en el área de control estadístico de la calidad, por medio del muestreo por aceptación y la inspección por atributos.

\section{REFERENCIAS}

[1] Department of Defense. "Military standard sampling procedures and tables for inspection by attributes”. USA. 1989.

[2] D. Bray and D. Lyonm. "Three class attributes plans in acceptance sampling". Technometrics. Vol. 13, Issue 3, pp. 575-585. April, 1973. ISSN: 0040-1706.

[3] C. Hobbs. "Inspection by attributes: A method of comparing rules for passing between normal and tightened inspection". International Journal of Quality and Reliability Management. Vol. 4, Issue 4, pp. 47-60. September, 1987. ISSN: 1757-2185.
[4] Department of Defense. "Military standard sampling procedures and tables for inspection by attributes". USA. 1963.

[5] H. Dodge. "A sampling inspection plan for continuous production. The Annals of Mathematical Statistics". Vol. 14, Issue 3, pp. 264-279. April, 1943. ISSN: 0020- 3157.

[6] T. Koyama. "Modified switching rules for sampling schemes such as MIL-STD-105D". Technometrics. Vol. 20, Issue 1, pp. 95-102. April, 1978. ISSN: 0040-1706.

[7] T. Koyama. "Switching characteristics under MIL-STD-105D”. Technometrics. Vol. 21, Issue 1, pp. 9-19. September, 1979. ISSN: 0040-1706.

[8] E. Shilling and J. Sheesley. "The performance of MIL-STD-105D under the switching rules part 1: Evaluation". Journal of Quality Technology. Vol. 10, Issue 2, pp. 76-83. May, 1978. ISSN: 0022-4065.

[9] W. Lou and J. Fu. "On probabilities for complex switching rules in sampling inspection. In: Scan Statistics and Methods and Applications". Statistics for Industry and Technology. Birkhäuser Boston. 23th edition. Vol. 1, pp. 203-219. Hamilton, Canada. September, 2009. ISBN: 978-0-8176-4748-3.

[10] R. Brugger. "Simplified Markov chain analysis of ANSI/ASQC Z1.4 used without limit numbers". Journal of Quality Technology. Vol. 21, Issue 2, pp. 97-102. September, 1989. ISSN: 0022-4065.

[11] S. Chakraborty and M. Bapaye. "Effect of inspection error on MIL-STD-105D sampling plans: Some observations". International Journal of Quality and Reliability Management. Vol. 6, Issue 2, pp. 60-72. May, 1989. ISSN: 1757-2185.

[12] G. Yang. "A renewal look and switching rules in the MIL-STD-105D sampling system". Journal of Applied Probability. Vol. 27, Issue 1, pp 183-192. November, 1990. ISSN: 0266-4763.

[13] G. Shumueli and A. Cohen. "Run-related probability functions applied to sampling inspection". Technometrics. Vol. 42, Issue 2, pp. 188-202. April, 2000. ISSN: 0040-1706. 